\title{
Breathing synchronized electrical stimulation of the abdominal muscles in patients with acute tetraplegia
}

\author{
T. Schauer ${ }^{1}$, R. Stephan ${ }^{1}$, A. Niedeggen ${ }^{2}$, T. Liebscher ${ }^{2}$, J. Dorien², R. O. Seidl ${ }^{2}$ \\ ${ }^{1}$ Technische Universität Berlin, Germany, schauer@control.tu-berlin.de \\ ${ }^{2}$ Unfallkrankenhaus Berlin, Germany
}

\section{Introduction}

A major concern in posttraumatic care of spinal cord injured (SCI) patients with a lesion at cervical and thoracic level (C5-Th3) is airway management and prevention of secondary medical complications. The aim of a DGUV funded research project is the development and clinical evaluation of an assistive system for supporting the process of weaning, for reducing the risk of pulmonary complications and for enabling increased cuffing.

\section{Methods}

Based on the principles of functional electrical stimulation (FES) the system was developed. Triggered by the measured air flow, stimulation of the abdominal muscles using surface electrodes supports the expiration in patients. The multichannel stimulator RehaStim2 (HASOMED GmbH) was extended by serial interfaces to two ventilation systems, Evita Infinity (Drägerwerk) and Elisée (ResMed Inc.), as well as to a spirometer (MicroLab-MK8, CareFusion Inc.). The flow is analysed in real-time, and the duration of stimulation inside the respiration phase is automatically adapted to the observed respiratory rate. Supported are complete and assisted ventilation as well as spontaneous breathing. Different training programs were developed which realise training phases with different stimulation intensities (for quiet breathing and coughing) and phases without stimulation (for rest and reference measurements).

\section{Results}

The developed system was successfully deployed at the Unfallkrankenhaus Berlin and is currently used in a clinical study with acute patients. Four patients have already used the system two times a day for 30 minutes every work day over 3 months. First results indicate at least for patients with spontaneous breathing an increase in peak flow and tidal volume.

\section{Conclusion}

A stimulation system for SCI patients with a high lesion was developed to support weaning. A study is still ongoing. The clinical outcome (pulmonary infections) will be investigated and compared to standard treatment based on retrospective patient data. 